\title{
Prevalence of respiratory diseases in relation to smoking rate in adults living in four Chinese cities: a comparison between 2017- 2018 and 1993-1996
}

\author{
Meilin Yan ${ }^{1}$, Jicheng Gong ${ }^{1}$, Qin Liu ${ }^{2}$, Wenyan $\mathrm{Li}^{2}$, Xiaoli Duan ${ }^{3}$, Suzhen Cao ${ }^{3}, \mathrm{Sai} \mathrm{Li}^{3}$, Lingyan $\mathrm{He}^{4}$, \\ Zixuan Yin ${ }^{4}$, Weiwei Lin ${ }^{5}$, Junfeng Jim Zhang ${ }^{1,6,7,8}$
}

${ }^{1}$ Beijing Innovation Center for Engineering Science and Advanced Technology, State Key Joint Laboratory for Environmental Simulation and Pollution Control, College of Environmental Sciences and Engineering, And Center for Environment and Health, Peking University, Beijing, China; ${ }^{2}$ School of Public Health and Management, Research Center for Medicine and Social Development, Collaborative Innovation Center of Social Risks Governance in Health, Chongqing Medical University, Chongqing, China; ${ }^{3}$ School of Energy and Environmental Engineering, University of Science and Technology Beijing, Beijing, China; ${ }^{4}$ Key Laboratory for Urban Habitat Environmental Science and Technology, School of Environment and Energy, Peking University Shenzhen Graduate School, Shenzhen, China; ${ }^{5}$ Department of Occupational and Environmental Health, School of Public Health, Sun Yat-Sen University, Guangzhou, China; ${ }^{6}$ Duke Kunshan University, Kunshan, China; ${ }^{7}$ Nicholas School of the Environment and Duke Global Health Institute, Duke University, Durham, USA; ${ }^{8}$ Guangzhou Institute of Respiratory Health, The First Affiliated Hospital of Guangzhou Medical University, Guangzhou, China

Contributions: (I) Conception and design: J Gong, M Yan, JJ Zhang; (II) Administrative support: J Gong; (III) Provision of study materials: J Gong, JJ Zhang; (IV) Collection and assembly of data: Q Liu, W Li, X Duan, S Cao, S Li, L He, Z Yin, W Lin; (V) Data analysis and interpretation: M Yan, J Gong; (VI) Manuscript writing: All authors; (VII) Final approval of manuscript: All authors.

Correspondence to: Jicheng Gong, PhD. College of Environmental Sciences and Engineering, Peking University, 5 Yiheyuan Road, Haidian, Beijing 100871, China. Email: jicheng.gong@pku.edu.cn.

Background: The sustained high prevalence of smoking in China has contributed substantially to the burden of chronic diseases, including respiratory diseases. This study compared the prevalence of smoking and respiratory diseases in Chinese adults between two time periods spanning over 25 years.

Methods: Cross-sectional surveys were performed in four Chinese cities of Chongqing, Lanzhou, Wuhan, and Guangzhou in 1993-1996 (Period 1) and in 2017-2018 (Period 2). Participants completed questionnaires asking smoking status, the presence of asthma and chronic bronchitis, education attainment and household characteristics. Logistic regression models were used to estimate the odds ratios of disease prevalence with regard to active smoking status for men and passive smoking status for women.

Results: Prevalence of asthma, prevalence of chronic bronchitis, and smoking rate, all decreased from Period 1 to Period 2. We observed strong evidence that active smoking increased prevalence for both asthma and chronic bronchitis in men during Period 1, with spatial heterogeneity and modifying effect by collegelevel education. Home exposure to passive smoking was associated with increased odds of having chronic bronchitis among female participants in Chongqing during Period 2, although the association was not statistically significant.

Conclusions: The prevalence for asthma and chronic bronchitis were lower in 2017-2018 compared to 25 years ago in the same four Chinese cities. Decreased smoking rate may have contribution to the improvement of these respiratory diseases. Male smokers, especially those without college-level education, showed higher prevalence of chronic bronchitis compared to nonsmokers during Period 1.

Keywords: Smoking; asthma; bronchitis

Submitted Feb 20, 2020. Accepted for publication May 20, 2020.

doi: 10.21037/jtd-19-crh-aq-002

View this article at: http://dx.doi.org/10.21037/jtd-19-crh-aq-002 


\section{Introduction}

Epidemiological studies have shown that both active smoking and passive smoking exposure (also referred to as environmental tobacco smoke) can be related to the onset and development of respiratory diseases and symptoms (1-5), with some evidence indicating that the association between cigarette smoking and asthma may be biologically plausible $(5,6)$. For example, cigarette smoking can pose a greater threat to asthmatic smokers than nonsmokers through accelerating decline in lung function, elevating induced sputum neutrophil counts, and impairing the effects of corticosteroids $(5,7)$. Passive smoking exposure is also adversely related to a variety of respiratory outcomes, including the frequent respiratory symptoms, lower respiratory infections, and lung cancer $(8,9)$, especially among susceptible populations such as women (1) and children (10).

Tobacco smoking exposure can also interact with other risk factors, such as individuals' demographic and socioeconomic characteristics, as well as other types of environmental exposure (e.g., household biomass combustion, ambient air pollution), in affecting respiratory health (1,11-15). For example, studies examining the link between smoking exposure and adults' respiratory symptoms found that the effect estimates varied between males and females $(1,11)$. A China-based study reported additive effects of passive smoking and coal heating on respiratory symptoms (15). A large-scale study in India found that smoking and use of unclear cooking fuels were associated with increased odds of asthma and chronic bronchitis among adults (14).

There are consistent evidence regarding substantial public health burden attributable to smoking in China $(16,17)$. Despite the implementation of smoking policy, the prevalence of smoking in China has remained high in the past decades (18-20). According to a national survey in 1996, about $63 \%$ of men were current smokers (19). The recent China Pulmonary Health study also reported a fairly high prevalence-51.2\% $(10,978 / 21,446)$ of male participants aged 20 years or older in 2015 were current smokers (20). Although the prevalence of asthma in China is generally lower than those in developed countries $(21,22)$, the large population of China results in a great number of patients suffering this illness, placing a considerable burden on families and the entire society. Furthermore, the prevalence of diagnosed asthma appears to be on rise in Chinese cities in recent years $(23,24)$. Taken together, the sustained high prevalence of smoking and the high frequency of respiratory disease make it important to examine the health effect of Chinese adults from exposure to smoking.

In the period of 1993-1996, an epidemiologic study was conducted to investigate the effects of outdoor air pollution and residential characteristics on respiratory health conditions among elementary school children (aged between 6 to 13) $(25,26)$. The study also collected information from parents of those children, including the information on their respiratory disease, smoking status, and education attainment. Because the study focused on children's respiratory health, no reports on adults' respiratory health outcomes have been published. Over the past two decades, most Chinese cities have experienced rapid urbanization and industrialization. The growth has been inevitably accompanied by the expansion of urban population, as well as substantial changes in people's living environment and lifestyle. To evaluate potential changes in respiratory disease prevalence, which may be associated with these rapid environmental and socioeconomical changes, we conducted another cross-sectional study in the same four cities from 2017 to 2018, collecting similar information on school children and parents using similar survey instrument. We term the 1993-1996 study as Period 1 study and the more recent study as Period 2 study. In this paper, we analyzed the data from both periods to examine associations of adults' respiratory diseases with active smoking, passive smoking, socioeconomic characteristics, and household characteristics.

\section{Methods}

\section{Questionnaire survey and participants}

The studies in both periods were conducted in four cities located in different regions of China, including Chongqing, Wuhan, Lanzhou, and Guangzhou. Chongqing, one of the major metropolitan cities in China, is located in the southwest region and has subtropical humid monsoon climate. It had a resident population of more than 31 million across the whole Chongqing municipality, among which about $64 \%$ were living in urban areas in 2017 (27). Wuhan, a major city in Central China, has subtropical monsoon climate, with annual average temperature of $17.3{ }^{\circ} \mathrm{C}$ in 2017 (28). There were about 11 million residents living on the $8,569.15 \mathrm{~km}^{2}$ land area in 2017 in Wuhan (28). Lanzhou is located in the northwest region of China and has temperate continental 
climate. The population of Lanzhou was over 3 million in 2017, with approximately $70 \%$ urban residents (29). Guangzhou, located in the southern coastline of China, has subtropical monsoon climate and an annual average temperature of $22^{\circ} \mathrm{C}$. Guangzhou is also one of the most densely-populated cities in China. It had a total area of $7,434.4 \mathrm{~km}^{2}$ and about 14 million residents in 2017 (30). In Period 1, one elementary school in an urban area and one school in a suburb were selected within each city. In Period 2, attempts were made to select the same schools or schools as close to the original schools as feasible. Because the school originally selected in the suburb of Guangzhou was closed, we selected a different school in a suburb of Shenzhen city, located about $140 \mathrm{~km}$ from Guangzhou. One school was selected from the urban area of each city in Period 2 except Wuhan where five schools in the urban area were included. The cross-sectional study in Period 2 was approved by the Institutional Review Board of Duke Kunshan University.

In both periods, the questionnaires were administered, face-to-face, by trained investigators. Within each household, one adult (father or mother) filled out a questionnaire, with help from investigators when necessary. Questionnaires were generally the same between the two periods, with modifications made to reflect certain conditions in Period 1 that were no long existent in Period 2. In Period 2, we asked general questions for each household characteristics about home heating, cooking with coal as the fuel and ventilation when cooking. In contrast, in Period 1, we asked these questions corresponding to three age ranges of children. To make the results from two surveys comparable, for each household in Period 1, we only considered the answer for a certain age range which contains the current age of child in that household. We approached in the same way for questions about cooking fuel and ventilation condition. Socioeconomic status was represented by education attainment. Multiple choice questions concerning education attainment were asked with different options in the two periods. To obtain consistent answers, we generated a dichotomized variable for education - whether college-level degree was achieved-by aggregating the multiple options. Data were not available for the exact age of participants, however, we assumed the two populations from both periods were comparable in terms of age distribution, because participants from Period 1 and 2 were both parents of children aged 6-13 and the age distributions among children from two periods were generally comparable (Figure S1).
We asked questions about smoking status and physiciandiagnosed asthma and chronic bronchitis for both male and female participants, separately. They were all "Yes" or "No" questions. We classified male participants (i.e., fathers of study children) as exposed to active smoking if answering "Yes" to the smoking-related questions. Since smoking among female participants (i.e., mothers of study children) were rare with an average prevalence of $1.2 \%$ across eight study districts (Table S1), we instead analyzed the effects of passive smoking at home on women's respiratory health to avoid potential model convergence problems. Female participants were defined as exposed to passive smoking at home if their husbands were classified as current smokers. Since we did not have detailed information of active smoking of husbands, we could not determine the exposure time or the frequency of passive smoke exposure for females in the study.

\section{Statistical analysis}

We primarily examined the overall associations between smoking exposure and respiratory diseases prevalence across the four Chinese cities during Periods 1 and 2, respectively. Univariate and multivariable logistic regression models were used; in the latter a set of models were fitted to examine potential confounding effects from both ambient environment and household characteristics. An indicator variable for city was included in the model. Within each city, we conducted surveys at schools in both urban and suburban districts, so an indicator variable denoting urban/suburban was included as well. Interaction terms were selected when they were statistically significant at $\alpha=0.15$. Based on that, the modifying effect of collegelevel education on the associations between smoking and respiratory diseases was examined. Odds ratios (ORs) for asthma and bronchitis associated with active smoking for males and passive smoking for females and the corresponding confidence intervals (CIs) were calculated based on the estimated coefficients from the regression models.

To investigate the city-specific variations in respiratory effects of smoking, we estimated the crude ORs and the adjusted ORs for each city, based on the fitted logistic regression models. Adjusted ORs were calculated from multivariable regression models with indicator variables for residential location (i.e., urban/suburban), home heating, cooking with coal, ventilation when cooking, and collegelevel attainment. In addition, home heating was not 
Table 1 Household and individual characteristics of study population in Period 1 (1993-1996) and Period 2 (2017-2018)

\begin{tabular}{|c|c|c|}
\hline Characteristics & Period 1 & Period 2 \\
\hline $\mathrm{N}$ & 1,452 & 2,126 \\
\hline Urban & $1,011(69.6)$ & $1,599(75.2)$ \\
\hline Heating & $441(30.4)$ & $619(29.1)$ \\
\hline Ventilation & 963 (66.3) & $2,111(99.3)$ \\
\hline College (males) & 309 (21.3) & $496(23.3)$ \\
\hline College (females) & $171(11.8)$ & $484(22.8)$ \\
\hline \multicolumn{3}{|l|}{ Wuhan, n (\%) } \\
\hline Heating & 842 (33.5) & $861(27.3)$ \\
\hline Cooking with coal & $890(35.4)$ & $142(4.5)$ \\
\hline Ventilation & $1,033(41.0)$ & $2,733(86.7)$ \\
\hline College (males) & $368(14.6)$ & $718(22.8)$ \\
\hline College (females) & $156(6.2)$ & $628(19.9)$ \\
\hline \multicolumn{3}{|l|}{ Lanzhou, n (\%) } \\
\hline $\mathrm{N}$ & 1,483 & 2,066 \\
\hline Urban & $716(48.3)$ & $848(41.0)$ \\
\hline \multicolumn{3}{|l|}{ Guangzhou, n (\%) } \\
\hline $\mathrm{N}$ & 2,302 & 2,306 \\
\hline Urban & $920(40.0)$ & $1,311(56.9)$ \\
\hline Cooking with coal & $157(6.8)$ & $28(1.2)$ \\
\hline Ventilation & $2,250(97.7)$ & 2,266 (98.3) \\
\hline College (males) & $921(40.0)$ & $1,118(48.5)$ \\
\hline College (females) & $603(26.2)$ & $1,097(47.6)$ \\
\hline
\end{tabular}

adjusted for Guangzhou and Lanzhou since home heating was generally not necessary in Guangzhou due to its warm climate. In contrast, over $95 \%$ of the study households in Lanzhou used home heating in both periods (Table 1).
Finally, we conducted a sensitivity analysis to examine the associations between household passive smoking exposure and female respiratory diseases excluding women who were also active smokers. All analyses were conducted in $\mathrm{R}$ 
Table 2 Prevalence of asthma and chronic bronchitis among males and females in Period 1 (1993-1996) and Period 2 (2017-2018)

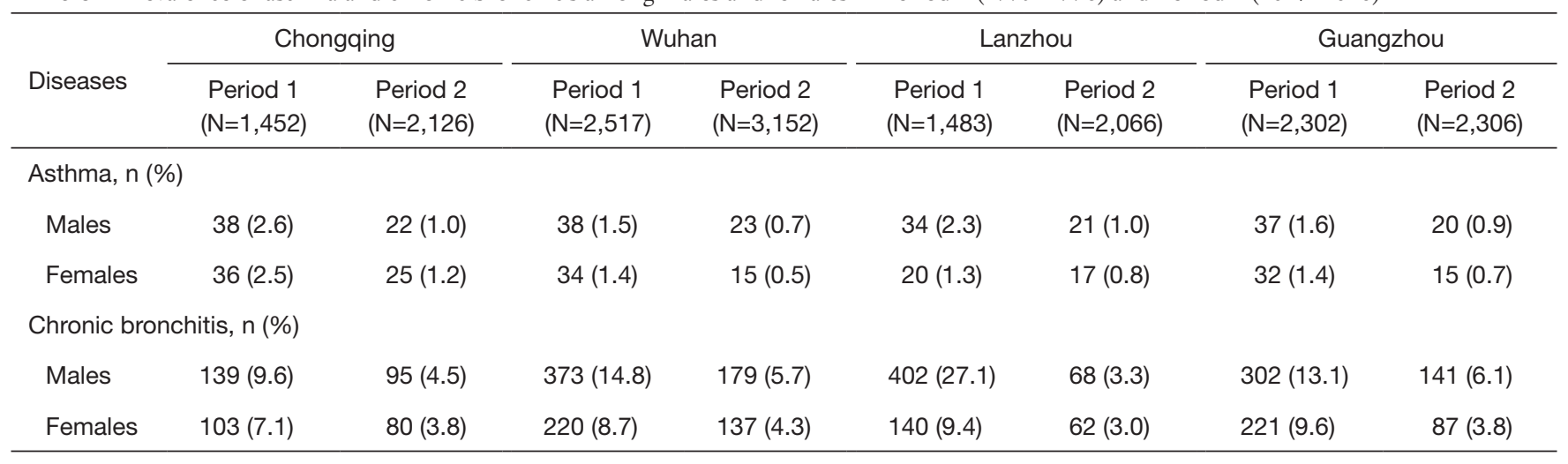

(version 3.4.2) (R Core Team 2017).

\section{Results}

\section{Study participants}

In Period 1, a total of 7,621 questionnaires were distributed among the selected schools in four cities; and 7,557 subjects returned the questionnaires, achieving an overall response rate of $99.2 \%$. During Period 2, we distributed a total of 11,449 questionnaires; and lost 9,974 non-respondents (87.1\% overall response rate).

Household and individual characteristics for all the participants in the four cities are shown in Table 1. An average of over 2,000 participants were included for a single study period of a given city. The proportions of participants from urban areas were fairly consistent between the two periods in the four cities. Ventilation during cooking had been generally improved from Period 1 to Period 2, for example, $41.0 \%$ households had cooking ventilation in Wuhan in Period 1 versus $86.7 \%$ in Period 2. In Lanzhou and Wuhan, more families used coal as cooking fuel in Period 1 than in Period 2 (e.g., $54.6 \%$ versus $1.9 \%$ in Lanzhou). The proportion of college-level education among participants generally increased from Period 1 to Period 2 in all the four cities. We observed little change in the prevalence of winter heating in the study households.

\section{Smoking exposure and respiratory disease prevalence}

Across the four study cities, the prevalence of asthma and chronic bronchitis in both males and females have generally halved from Period 1 to Period 2, with the largest change observed for male chronic bronchitis prevalence in
Lanzhou-27.1\% versus 3.3\% (Table 2). The prevalence of asthma for males and females were very close by city and study period. In contrast, chronic bronchitis was more common in male participants than those in females. For example, in Period 1, the prevalence were $14.8 \%$ and $8.7 \%$ for males and females in Wuhan, respectively.

Table 3 demonstrated the prevalence of respiratory diseases associated with active smoking in males and passive smoking in females. Across the four cities, the smoking rates had decreased from Period 1 to Period 2. In Chongqing, while $76.7 \%(1,113$ out of 1,452$)$ of the households reported smoking exposure at home in Period $1,44.0 \%$ (936 out of 2,126$)$ reported smoking in Period 2. This decline was accompanied by a substantial reduction in the crude prevalence of both asthma and chronic bronchitis among male participants (Table 3), especially in Lanzhou (bronchitis prevalence in males: $32.0 \%$ in Period 1 versus $3.0 \%$ in Period 2). Furthermore, chronic bronchitis showed a higher prevalence than asthma among the study subjects. For example, in Guangzhou during Period 1, 13.5\% male smokers reported having chronic bronchitis and $1.5 \%$ had asthma (Table 3).

\section{Effects of smoking on respiratory disease prevalence across four cities}

Across the four cities, active smoking was negatively associated with asthma among male participants in Period 1 , though the association was not statistically significant (Table 4, M1-M4). Active smoking was associated with the prevalence of bronchitis in both periods, with the effect size attenuated in Period 2: crude OR of 1.79 (95\% CI, $1.52-2.10)$ in Period 1 versus 1.17 (95\% CI, 0.97-1.41) in Period 2. Further, we observed an appreciable modifying 


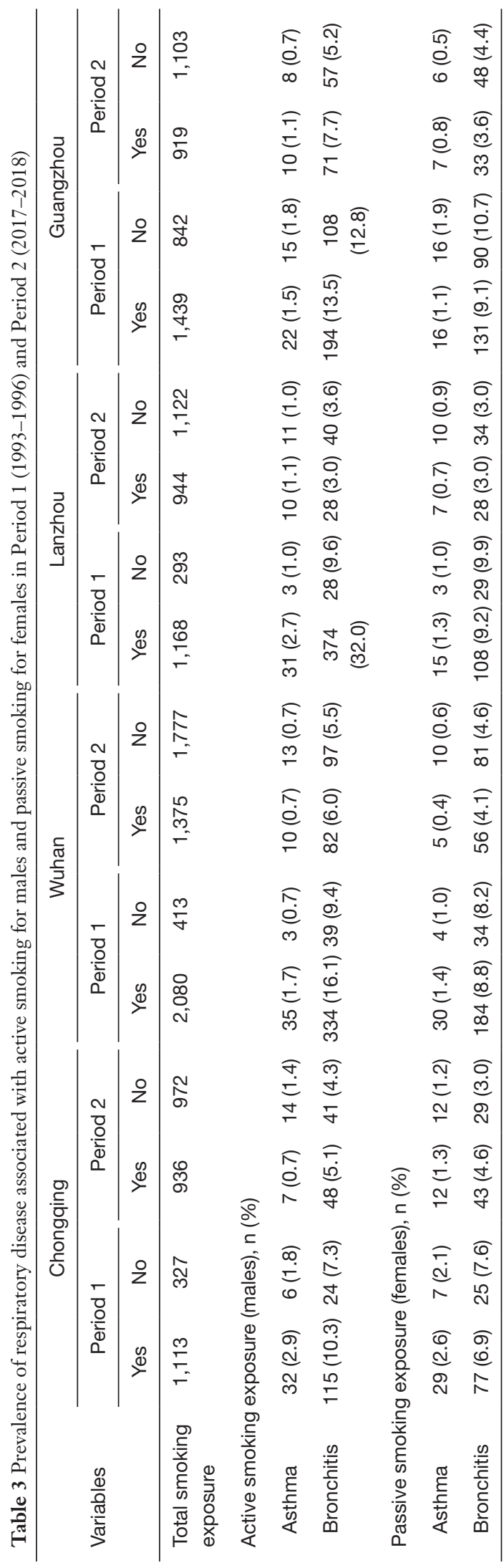

effect of educational level on active smoking-respiratory disease associations, especially for chronic bronchitis (Table 4, M5, M6). The male participants with lower-thancollege education showed much greater ORs of active smoking for chronic bronchitis compared with those with a college degree or above in Period 1, however, the difference was no longer clear in Period 2. In females, in contrast, associations between household passive smoking and the prevalence of asthma were only observed among those without college-level degree in both periods (Table 4).

\section{Effects of smoking on respiratory disease prevalence for each city}

Significant effects of active smoking on the prevalence of asthma and chronic bronchitis were observed among male participants in Chongqing, Wuhan, and Lanzhou in Period 1 but not in Period 2 (Figure 1). The largest ORs in the males during Period 1 were observed in Lanzhou: crude ORs of 2.64 (95\% CI, 0.80-8.68) and 4.46 (95\% CI, 2.96-6.71) for asthma and chronic bronchitis, respectively. Adjustment of potential covariates considered had little influence on the effect estimates. For example, the multivariable logistic regression analysis confirmed an OR of 1.67 (95\% CI, 1.04-2.67) for males' chronic bronchitis in Chongqing in Period 1, which was fairly consistent with the crude OR of 1.45 (95\% CI, 0.92-2.29). In Guangzhou, the odds of having asthma or chronic bronchitis in male smokers were more evident in Period 2 than in Period 1. After adjusting for the potential confounding variables, we estimated the active smoking associated ORs of 1.68 (95\% CI, 0.64-4.40) for asthma and 1.72 (95\% CI, 1.18-2.50) for chronic bronchitis among male respondents in Guangzhou during Period 2 (Figure 1).

The odds of having asthma in females exposed to passive smoking at home decreased from Period 1 to Period 2 in Chongqing, Wuhan, and Lanzhou, while increased in Guangzhou. We estimated the adjusted ORs to be 0.60 (95\% CI: 0.30-1.21) in Period 1 and 1.50 (95\% CI: 0.50-4.60) in Period 2 in Guangzhou. Negative associations between passive smoking and chronic bronchitis were most apparent in females of Chongqing during Period 2 (adjusted OR $=1.55$, 95\% CI: 0.96-2.50). Again, no significant impact of covariates on the effect estimates was observed in females. Although the statistical significance was not met in the estimates for females, the noticeable magnitude of the ORs in some estimates could suggest adverse effects of the passive smoking on females' respiratory disease prevalence. 
Table 4 Odds ratios (95\% confidence intervals) for asthma and bronchitis associated with active smoking for males and passive smoking for females across four cities in Period 1 and Period 2

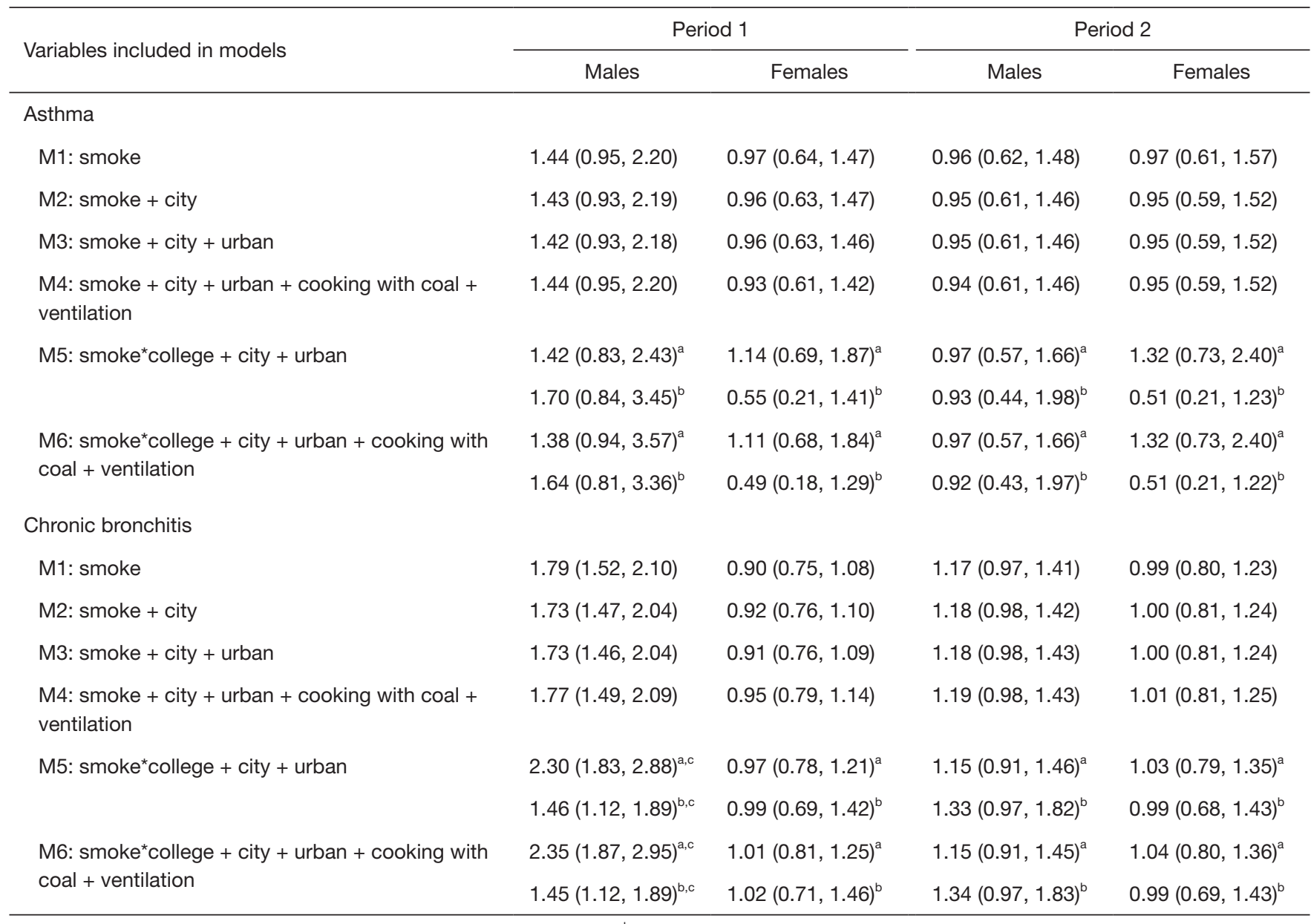

${ }^{a}$, odds ratio for males/females with no college-level education; ${ }^{b}$, odds ratio for males/females with college-level education or above; ${ }^{\text {, }}$ interaction terms in these models are statistically significant $(\mathrm{P}<0.01)$.

Sensitivity analysis for the female non-smokers indicated the robustness of our primary results for Chongqing, Wuhan, and Lanzhou (Figures 2,S2). However, after excluding female smokers in Guangzhou, ORs for the passive smoking and respiratory diseases in Period 2 were greater than those in the primary analysis, though the ORs in Period 1 were consistent with our primary results (Figure S3).

\section{Discussion}

In the current analysis, we examined the effects of tobacco smoking on the respiratory diseases among adults in four Chinese cities during two cross-sectional surveys (1993-1996 versus 2017-2018). Household and individual characteristics were changed across the two study periods, as evidenced by appreciable changes in the proportions of using coal as the cooking fuel, cooking ventilation condition, and the college-degree attainment. Smoking rate and prevalence of asthma and chronic bronchitis all decreased from the 1993-1996 period (Period 1) to the 2017-1018 period (Period 2). We observed strong evidence of adverse effects of active smoking on the prevalence of males' asthma and chronic bronchitis during Period 1, with spatial heterogeneity and modifying effect of college-level education. Passive smoking at home was associated with the increased odds of having chronic bronchitis among females in Chongqing during Period 2, though the association was not statistically significant.

Our finding of adverse effects of active smoking on the respiratory diseases among males in four Chinese 


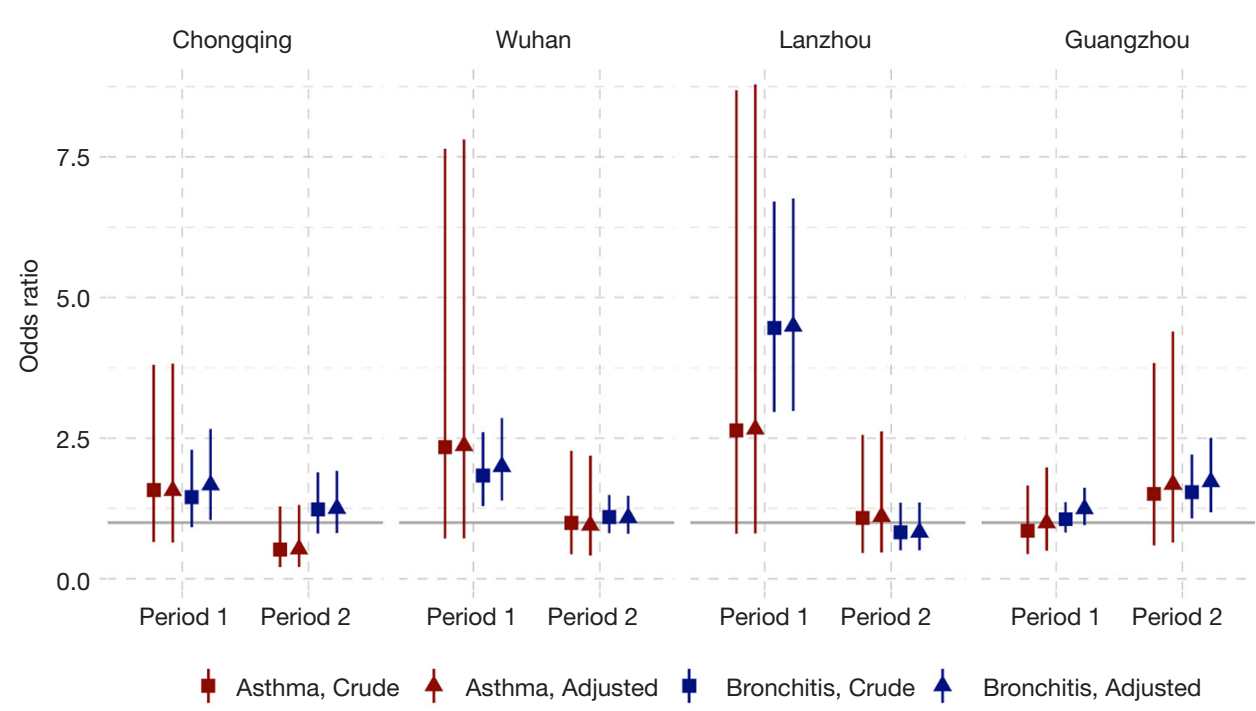

Figure 1 Crude and adjusted odds ratio (OR) and 95\% confidence intervals for asthma and bronchitis among males associated with selfreported active smoking in Period 1 and Period 2. Adjusted ORs were estimated from regression models controlling location, college-level education, home heating (only adjusted for Chongqing and Wuhan), cooking with coal as fuel, and cooking ventilation.

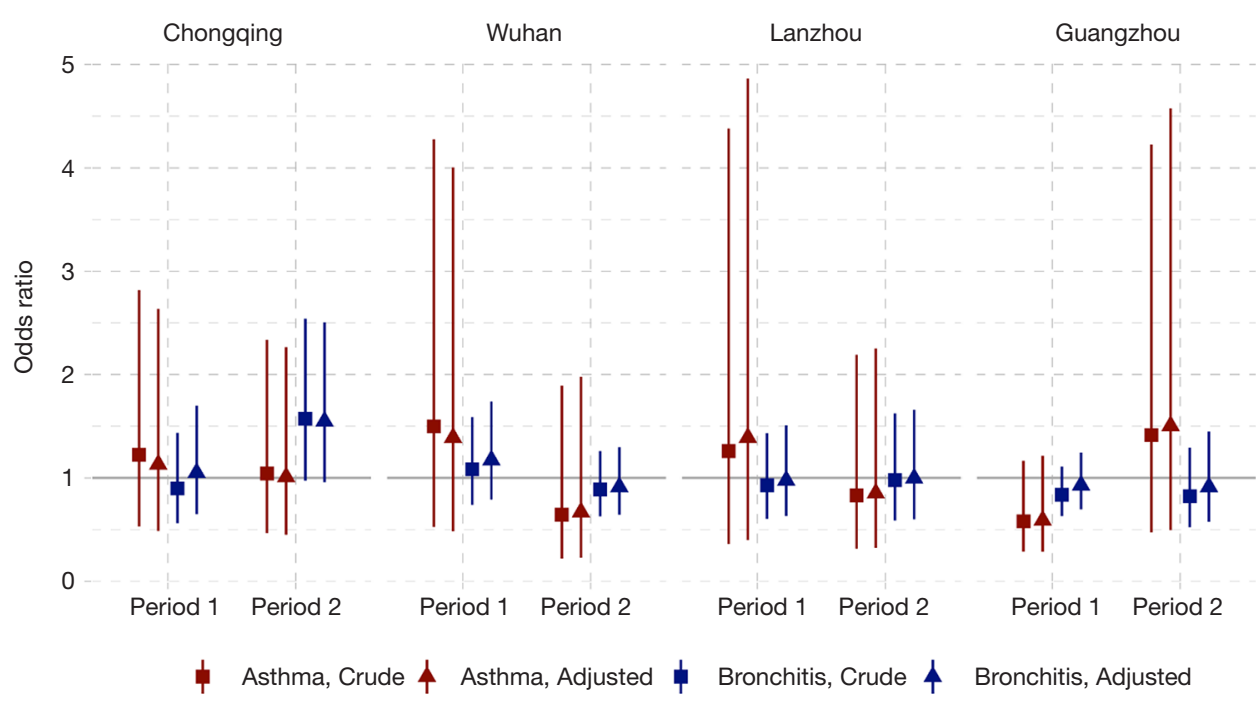

Figure 2 Crude and adjusted odds ratio and $95 \%$ confidence intervals for asthma and bronchitis among females associated with passive smoking exposure at home in Period 1 and Period 2. Adjusted ORs were estimated from regression models controlling location, collegelevel education, home heating (only adjusted for Chongqing and Wuhan), cooking with coal as fuel, and cooking ventilation.

cities was in line with those from earlier studies $(3,8,31)$, including some conducted among Chinese populations $(32,33)$. A meta-analysis study reported a risk ratio of 1.61 (95\% CI, 1.07-2.42) for asthma for smokers than for never smokers (8). Another worldwide meta-analysis estimated a relative risk of 3.41 (95\% CI, 3.13-3.72) for chronic bronchitis for current smokers of any tobacco products in reference to non-smokers (31). Furthermore, while we estimated heightened effects of active smoking on respiratory diseases of males in Period 1, those associations were no longer clear during Period 2 in the same study areas. Several factors might help to explain the difference in 
the estimated effects from two periods. First, the prevalence of both smoking exposure and diseases were higher in Period 1 than Period 2. Second, unmeasured confounding factors might be possible in our analysis, especially for Period 1 when pronounced effects were observed. For example, ambient air pollution levels were significantly associated with several respiratory health outcomes (e.g., asthma and bronchitis) in Chinese cities (34). Ambient pollution could have been an important confounder. Given that the ambient air pollution has been improved over the past decade, our estimates for Period 1 may be more likely to be confounded by the ambient air pollution than those for Period 2. Finally, in Period 2, more participants have used more clean fuel and improved ventilation for cooking. The association between smoking and the prevalence of respiratory diseases may still be somewhat confounded by the greatly improved indoor air pollution from cooking, despite that adjusting these variables in the analysis resulted in little difference of smoking effects observed in the crude model.

When analyzing the interaction of smoking status and education level, we observed substantially increased effects in the prevalence of bronchitis among males who did not obtain the college-level education in the Period 1. Participants without a college-level education appeared more likely to be blue-collar workers, thus they were possibly exposed to allergen of respiratory disease from their occupational environment. For example, a cross-sectional survey conducted in rural Beijing, China, provided evidence that occupational exposure to insecticides and fertilizers was significantly associated with increased reporting of respiratory symptoms (32).

We did not observe significant associations between increased prevalence of asthma and chronic bronchitis and passive smoking at home in female participants. Some previous studies reported increased risk of asthma among passive smokers $(1,35)$. For example, a meta-analysis of longitudinal studies reported passive smoking exposure was associated with the increased risk of lung cancer in adult nonsmokers (8). In this study, with a categorical exposure variable, it might be difficult to capture the overall health effect of the passive smoking exposure. One prospective study measured the passive smoking exposure in previous week using personal nicotine badge, and found that the intensity of exposure was associated with the exacerbated consequences of asthma (36). Also, some studies found that the passive smoking at workplace could be more strongly associated with respiratory symptoms than at home
$(1,35,37,38)$. However, we could not assess subjects' passive smoking exposure outside homes due to the lack of data.

We found inconsistency in the estimated associations between passive smoking and the respiratory diseases of females in Guangzhou during the Period 2, regardless including the data of female smokers or not (Figure S3). This might be caused by the inclusion of a substitutional school from Shenzhen. Among the participants of the study, the smoking rate in females were much lower in Guangzhou [0.7\% $(10 / 1,382)$ in suburban area in Period 1] than that in Shenzhen [7\% (70/995)] (Table S1). There were 70 female smokers in Shenzhen (Table S1, the right bottom cell), among which 20 were classified as unexposed to passive smoking at home because their husbands were nonsmokers (data not shown). However, those 20 females were essentially exposed to smoke because they themselves were smokers. Thus, the misclassification of the passive smoking exposure could potentially bias our primary results toward the null (Figure S3).

In most cases, the adjusted ORs were similar to the crude ORs, indicating little confounding effect from the covariates considered in this study. Current findings of the interplay between smoking and some potential confounders (e.g., demographical and socioeconomic factors) in affecting adults' respiratory health is still conflicting. The Swiss Study on Air Pollution and Lung Diseases in Adults found that the effect of passive smoking exposure on asthma was not influenced by gender, education, and passive smoking exposure history in childhood (35). Another study conducted in Finland, Estonia, and Sweden reported that the odds of chronic bronchitis associated with exposure to the passive smoking in females were more pronounced than those in males (1). Although the inconsistency might stem from the different methods of addressing confounding effects, further studies are needed to explore how potential confounders may affect the associations between the smoking exposure and respiratory health outcomes.

In examining the respiratory effects of passive smoking at home, females were deemed to be exposed if their husbands reported as current smokers. This definition of the passive smoking exposure, which has also been used in earlier studies (39), could reduce the likelihood of information bias. With self-reporting passive smoking status, subjects with respiratory diseases tended to attribute their disease to smoking and reported more exposure than they actually received, as compared with non-diseased subjects.

Our study has several limitations. Because the primary goal of the studies in both periods was to assess potential 
risk factors of respiratory diseases for children rather than for adults, the data collected on adults were rather limited, including information on other potential confounding variables (e.g., age, childhood smoking exposure, nonhousehold passive smoking exposure, and whether subject was a former smoker or not) as suggested by previous studies $(4,35,40)$. With an assumption that the age distribution among adults in Period 1 and 2 could be very similar to each other, as both were parents of children aged 6 to 13 , we could compare the disease prevalence across two study periods. However, without exact age of study subjects, it will be hard to compare the disease prevalence presented in this study to others. In addition, we could assess neither the amount nor the duration of smoking exposure due to questionnaire limitations on smoking exposure assessment, though previous studies have showed doseresponse associations using quantitative smoking exposure measurements $(1,36)$. Thus, cautions should be taken when comparing our results with those estimated for another population whose smoking amount or duration is different from our study population. Given the above limitations, the residual confounding effect may occur in our results due to either inadequate adjustments of confounders or measurement errors.

In conclusion, the prevalence for two major respiratory diseases (asthma and chronic bronchitis) was reduced in parents of elementary students in four Chinese cities from the period of 1993-1996 to the period of 2017-2018. Male smokers, especially those without a college-level education, reported much higher chronic bronchitis prevalence compared with male non-smokers during the Period 1 . The smoking rate was low in female participants whose passive smoking exposure in homes was associated with increased prevalence of chronic bronchitis in Chongqing during Period 2 but not across the four cities.

\section{Acknowledgments}

Funding: Fund support from Beijing Innovation Center for Engineering Science and Advanced Technology (BICESAT).

\section{Footnote}

Provenance and Peer Review: This article was commissioned by the Guest Editors (Junfeng Zhang, Howard Kipen and Haidong Kan) for the series "Children's Respiratory Health and Air Quality" published in Fournal of Thoracic Disease.
The article was sent for peer review organized by the Guest Editors and the editorial office.

Data Sharing Statement: Available at http://dx.doi. org/10.21037/jtd-19-crh-aq-002

Conflicts of Interest: All authors have completed the ICMJE uniform disclosure form (available at http://dx.doi. org/10.21037/jtd-19-crh-aq-002). The series “Children's Respiratory Health and Air Quality" was commissioned by the editorial office without any funding or sponsorship. JJZ served as the unpaid Guest Editor of the series. JG and JJZ serves as editorial board members of Fournal of Thoracic Disease. The other authors have no other conflicts of interest to declare.

Ethical Statement: The authors are accountable for all aspects of the work in ensuring that questions related to the accuracy or integrity of any part of the work are appropriately investigated and resolved. The study was conducted in accordance with the Declaration of Helsinki (as revised in 2013). Approval of study protocol was obtained from Duke Kunshan University IRB (No. FWA00021580). Informed consent was obtained from parents or guardians of participating children.

Open Access Statement: This is an Open Access article distributed in accordance with the Creative Commons Attribution-NonCommercial-NoDerivs 4.0 International License (CC BY-NC-ND 4.0), which permits the noncommercial replication and distribution of the article with the strict proviso that no changes or edits are made and the original work is properly cited (including links to both the formal publication through the relevant DOI and the license). See: https://creativecommons.org/licenses/by-nc-nd/4.0/.

\section{References}

1. Larsson ML, Loit H, Meren M, et al. Passive smoking and respiratory symptoms in the FinEsS study. Eur Respir J 2003;21:672-6.

2. Knight A, Breslin AB. Passive cigarette smoking and patients with asthma. Med J Aust 1985;142:194-5.

3. Menezes AM, Victora CG, Rigatto M. Chronic bronchitis and the type of cigarette smoked. Int J Epidemiol 1995;24:95-9.

4. Vineis P, Airoldi L, Veglia F, et al. Environmental tobacco smoke and risk of respiratory cancer and chronic 
obstructive pulmonary disease in former smokers and never smokers in the EPIC prospective study. BMJ 2005;330:277.

5. Tamimi A, Serdarevic D, Hanania NA. The effects of cigarette smoke on airway inflammation in asthma and COPD: Therapeutic implications. Respir Med 2012;106:319-28.

6. Weiss ST, Utell MJ, Samet JM. Environmental tobacco smoke exposure and asthma in adults. Environ Health Perspect 1999;107:891-5.

7. Chalmers GW, MacLeod KJ, Thomson L, et al. Smoking and airway inflammation in patients with mild asthma. Chest 2001;120:1917-22.

8. Jayes L, Haslam PL, Gratziou CG, et al. SmokeHaz: systematic reviews and meta-analyses of the effects of smoking on respiratory health. Chest 2016;150:164-79.

9. Ho SY, Lam TH, Chung SF, et al. Cross-sectional and prospective associations between passive smoking and respiratory symptoms at the workplace. Ann Epidemiol 2007;17:126-31.

10. Vanker A, Gie R, Zar H. The association between environmental tobacco smoke exposure and childhood respiratory disease: A review. Expert Rev Respir Med 2017;11:661-73.

11. Lam TH, Ho LM, Hedley AJ, et al. Environmental tobacco smoke exposure among police officers in Hong Kong. JAMA 2000;284:756-63.

12. Reddy TS, Guleria R, Sinha S, et al. Domestic cooking fuel and lung functions in healthy non-smoking women. Indian J Chest Dis Allied Sci 2004;46:85-90.

13. Gupta D, Aggarwal A, Chaudhry K, et al. Household environmental tobacco smoke exposure, respiratory symptoms and asthma in non-smoker adults: A multicentric population study from India. Indian J Chest Dis Allied Sci 2006;48:31-6.

14. Jindal SK, Aggarwal A, Gupta D, et al. Indian study on epidemiology of asthma, respiratory symptoms and chronic bronchitis in adults (INSEARCH). Int J Tuberc Lung Dis 2012;16:1270-7.

15. Pope CA 3rd, Xu X. Passive cigarette smoke, coal heating, and respiratory symptoms of nonsmoking women in China. Environ Health Perspect 1993;101:314-6.

16. Gu D, Kelly TN, Wu X, et al. Mortality attributable to smoking in China. N Engl J Med 2009;360:150-9.

17. Gan Q, Smith KR, Hammond SK, et al. Disease burden of adult lung cancer and ischemic heart disease from passive tobacco smoking in China. Tob Control 2007;16:417-22.

18. Zhang J, Ou JX, Bai CX. Tobacco smoking in China: prevalence, disease burden, challenges and future strategies. Respirology 2011;16:1165-72.

19. Yang G, Fan L, Tan J, et al. Smoking in China: Findings of the 1996 national prevalence survey. JAMA 1999;282:1247-53.

20. Wang C, Xu J, Yang L, et al. Prevalence and risk factors of chronic obstructive pulmonary disease in China (the China Pulmonary Health [CPH] study): A national crosssectional study. Lancet 2018;391:1706-17.

21. To T, Stanojevic S, Moores G, et al. Global asthma prevalence in adults: Findings from the cross-sectional world health survey. BMC Public Health 2012;12:204.

22. Masoli M, Fabian D, Holt S, et al. The global burden of asthma: Executive summary of the GINA Dissemination Committee report. Allergy 2004;59:469-78.

23. Wang WY, Lin JT, Su N, et al. Survey on the prevalence rate of bronchial asthma in Beijing area among the residents aged over 14 years from 2010 to 2011. Zhonghua Yi Xue Za Zhi 2013;93:1383-7.

24. Zhang F, Hang J, Zheng B, et al. The changing epidemiology of asthma in Shanghai, China. J Asthma 2015;52:465-70.

25. Zhang JJ, Hu W, Wei F, et al. Children's respiratory morbidity prevalence in relation to air pollution in four Chinese cities. Environ Health Perspect 2002;110:961.

26. Qian Z, Zhang J, Wei F, et al. Long-term ambient air pollution levels in four Chinese cities: Inter-city and intracity concentration gradients for epidemiological studies. J Expo Anal Environ Epidemiol 2001;11:341.

27. Chongqing Bureau of Statistics. Chongqing Statistical Yearbook [Internet]. 2018. Available online: http://www. cqtj.gov.cn/tjnj/2018/indexch.htm

28. Wuhan Bureau of Statistics. Wuhan Statistical Yearbook [Internet]. 2018. Available online: http://tjj.hubei.gov.cn/ info/iList.jsp?cat_id=10436

29. Lanzhou Bureau of Statistics. Lanzhou Statistical Yearbook [Internet]. 2018. Available online: http://tjj.lanzhou.gov. cn/art/2018/11/29/art_4866_526974.html

30. Guangzhou Statistics Bureau. Population distribution of Guangzhou in 2017 [Internet]. 2018. Available online: http://www.gzstats.gov.cn/gzstats/index.shtml

31. Forey BA, Thornton AJ, Lee PN. Systematic review with meta-analysis of the epidemiological evidence relating smoking to COPD, chronic bronchitis and emphysema. BMC Pulm Med 2011;11:36.

32. Zhang LX, Enarson DA, He GX, et al. Occupational and environmental risk factors for respiratory symptoms in rural Beijing, China. Eur Respir J 2002;20:1525-31. 
33. Wilson D, Takahashi K, Pan G, et al. Respiratory symptoms among residents of a heavy-industry province in China: Prevalence and risk factors. Respir Med 2008;102:1536-44.

34. Li Y, Wang W, Kan H, et al. Air quality and outpatient visits for asthma in adults during the 2008 Summer Olympic Games in Beijing. Sci Total Environ 2010;408:1226-7.

35. Leuenberger P, Schwartz J, Ackermann-Liebrich U, et al. Passive smoking exposure in adults and chronic respiratory symptoms (SAPALDIA Study). Swiss Study on Air Pollution and Lung Diseases in Adults, SAPALDIA team. Am J Respir Crit Care Med 1994;150:1222-8.

36. Eisner MD, Klein J, Hammond SK, et al. Directly measured second hand smoke exposure and asthma health outcomes. Thorax 2005;60:814-21.

Cite this article as: Yan M, Gong J, Liu Q, Li W, Duan X, Cao S, Li S, He L, Yin Z, Lin W, Zhang JJ. Prevalence of respiratory diseases in relation to smoking rate in adults living in four Chinese cities: a comparison between 2017-2018 and 1993-1996. J Thorac Dis 2020;12(10):6315-6326. doi: 10.21037/jtd19-crh-aq-002
37. Janson C, Chinn S, Jarvis D, et al. Effect of passive smoking on respiratory symptoms, bronchial responsiveness, lung function, and total serum $\operatorname{IgE}$ in the European Community Respiratory Health Survey: A cross-sectional study. Lancet 2001;358:2103-9.

38. Janson C. The effect of passive smoking on respiratory health in children and adults [State of the Art]. Int J Tuberc Lung Dis 2004;8:510-6.

39. Kurahashi N, Inoue M, Liu Y, et al. Passive smoking and lung cancer in Japanese non-smoking women: A prospective study. Int J Cancer 2008;122:653-7.

40. Larsson ML, Frisk M, Hallström J, et al. Environmental tobacco smoke exposure during childhood is associated with increased prevalence of asthma in adults. Chest 2001;120:711-7. 


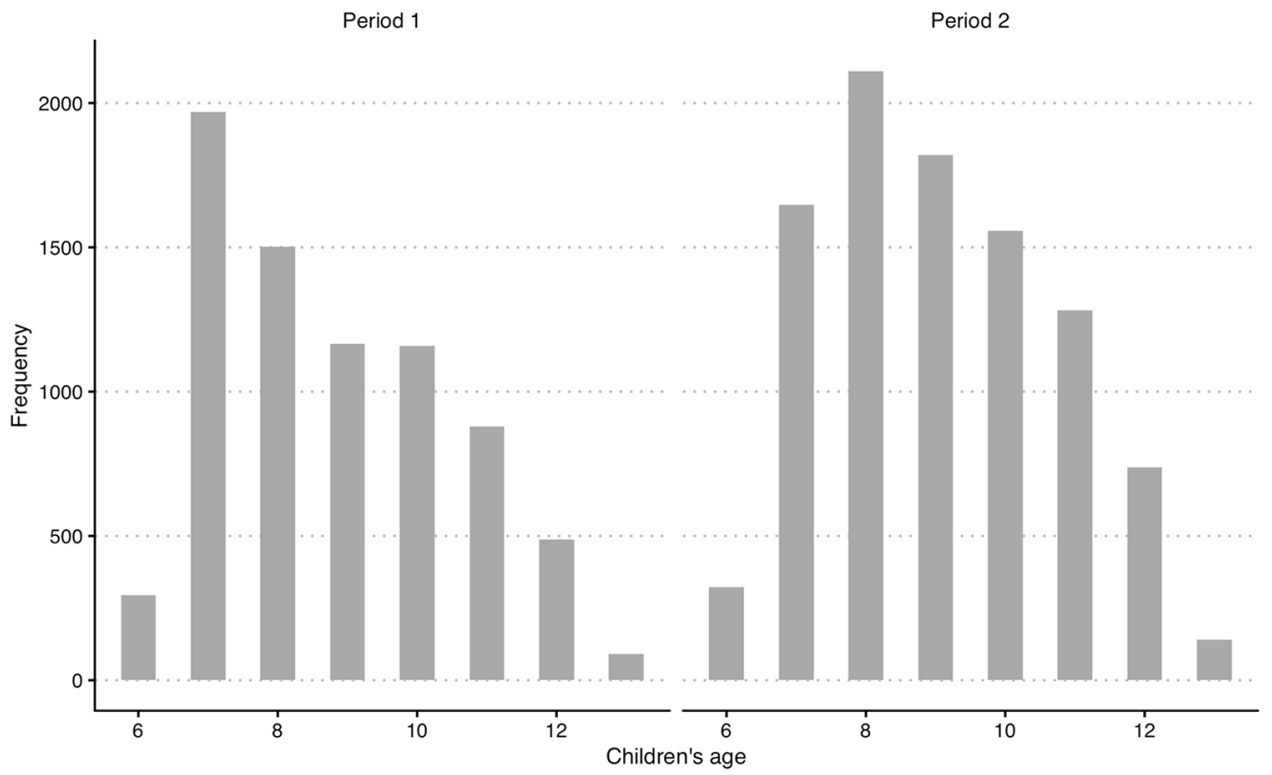

Figure S1 Age distributions for surveyed children across the four cities in Period 1 and Period 2. Surveyed children's father and mother are the participants in this study.

Table S1 Smoking prevalence in females in Period 1 (1993-1996) and Period 2 (2017-2018)

\begin{tabular}{|c|c|c|c|c|c|c|c|c|}
\hline Period & \multicolumn{2}{|c|}{ Chongqing } & \multicolumn{2}{|c|}{ Wuhan } & \multicolumn{2}{|c|}{ Lanzhou } & \multicolumn{2}{|c|}{ Guangzhou } \\
\hline \multicolumn{9}{|l|}{ Period 1} \\
\hline Urban & 1,011 & $24(2.4)$ & 593 & $8(1.3)$ & 716 & $6(0.8)$ & 920 & $13(1.4)$ \\
\hline Suburban & 441 & $5(1.1)$ & 1,924 & $30(1.6)$ & 767 & $3(0.4)$ & 1,382 & $10(0.7)$ \\
\hline Urban & 1,599 & $16(1.0)$ & 1,985 & $15(0.8)$ & 848 & $2(0.2)$ & 1,311 & $1(0.1)$ \\
\hline Suburban & 527 & $6(1.1)$ & 1,167 & $8(0.7)$ & 1,218 & $5(0.4)$ & 995 & $70(7.0)$ \\
\hline
\end{tabular}




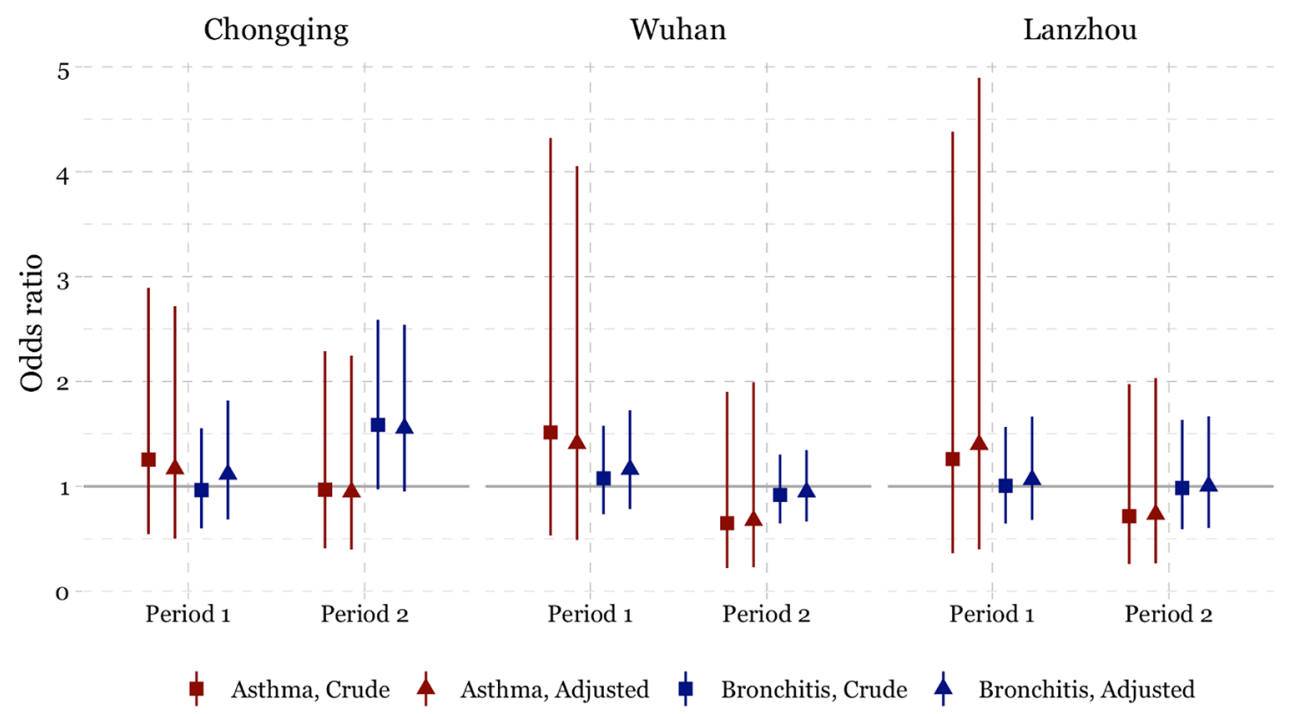

Figure S2 Crude and adjusted odds ratio and 95\% confidence intervals for asthma and bronchitis among females in Chongqing, Wuhan, and Lanzhou associated with household passive smoking in Period 1 and Period 2. Results were estimated based on models fit using data omitting female smokers.

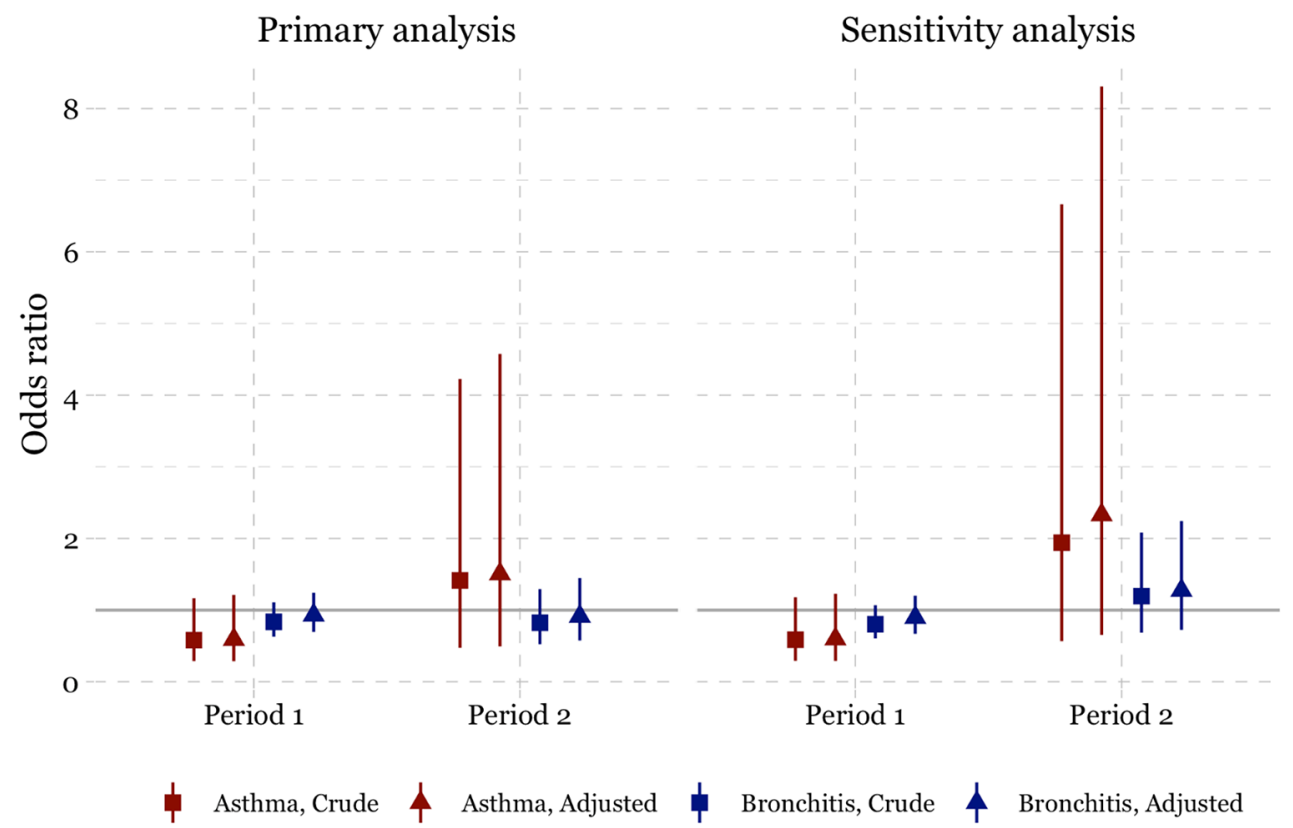

Figure S3 Crude and adjusted odds ratio and 95\% confidence intervals for physician-diagnosed asthma and bronchitis among females in Guangzhou associated with household passive smoking in Period 1 and Period 2. Results for "Primary analysis" were the same as shown in Figure 2. Results for "Sensitivity analysis" were estimated based on models fit using data omitting female smokers. 